\title{
A Biological Model for Resource Allocation and User Dynamics in Virtualized HetNet
}

\author{
Lu Ma $\mathbb{D}^{1,2}$ Xiangming Wen, ${ }^{1,2}$ Luhan Wang, ${ }^{1,2}$ Zhaoming Lu $\mathbb{D}{ }^{1,2}$ \\ Raymond Knopp, ${ }^{3}$ and Irfan Ghauri ${ }^{3}$ \\ ${ }^{1}$ Beijing Key Laboratory of Network System Architecture and Convergence, Beijing University of Posts and Telecommunications, \\ Beijing 100876, China \\ ${ }^{2}$ Beijing Advanced Innovation Center for Future Internet Technology, Beijing 100124, China \\ ${ }^{3}$ Communication System Department, EURECOM, 06410 Biot, France
}

Correspondence should be addressed to Lu Ma; malu@bupt.edu.cn

Received 20 April 2018; Revised 14 August 2018; Accepted 9 September 2018; Published 27 September 2018

Guest Editor: Dejan Vukobratovic

Copyright (C) $2018 \mathrm{Lu} \mathrm{Ma}$ et al. This is an open access article distributed under the Creative Commons Attribution License, which permits unrestricted use, distribution, and reproduction in any medium, provided the original work is properly cited.

\begin{abstract}
Virtualization technology is considered an effective measure to enhance resource utilization and interference management via radio resource abstraction in heterogeneous networks (HetNet). The critical challenge in wireless virtualization is virtual resource allocation on which substantial works have been done. However, most existing researches on virtual resource allocation focus on improving total utility. Different from the existing works, we investigate the dynamic-aware virtual radio resource allocation in virtualization based HetNet considering utility and fairness. A virtual radio resource management framework is proposed, where the radio resources of different physical networks are virtualized into a virtual resource pool and mobile virtual network operators (MVNOs) compete for virtual resources from the pool to provide service to users. A virtual radio resource allocation algorithm based on biological model is developed, considering system utility, fairness, and dynamics. Simulation results are provided to verify that the proposed virtual resource allocation algorithm not only converges within a few iterations, but also achieves a better tradeoff between total utility and fairness than existing algorithm. Besides, it can also be utilized to analyze the population dynamics of system.
\end{abstract}

\section{Introduction}

Within the last decade, mobile networks are experiencing dramatic increases in data traffic and services [1]. Mobile network operators deploy many kinds of networks and make them denser and denser. For example, in LTE, heterogeneous networks allow a mixed deployment of macro and micro base stations (BSs) in a geographic area providing different accessing capabilities and capacity/coverage needs [2]. However, chaotic and dense deployments cause new problems [3]. First, clients of one BS suffer high interference from neighboring BSs because of the high frequency reuse and broadcast nature of wireless communication. Second, coverage of cellular networks becomes denser and more complex which leads to more users at the edge of network. More handovers and unbalanced load are caused by user mobility. As a result, the benefit in terms of capacity of heterogeneous networks could be undermined if effective measures are not taken [4]. The wireless network virtualization which attracts much attention has recently been considered as a promising solution to increase the spectrum and infrastructure efficiency [5].

With virtualization technology, the wireless network infrastructure can be decoupled from services it provides so that different services can share the same infrastructure [6]. In wireless virtualization, the physical radio resources of heterogeneous networks owned by Infrastructure Providers (InPs) are abstracted and sliced into virtual radio resources and form a virtual radio resource pool [7]. Mobile virtual network operators (MVNOs) lease the virtual radio resources and assign them to users [8]. Since the infrastructure and physical resources of HetNet are abstracted and sliced into virtual resource, many effective measures could be carried out more easily, such as interference management, load balancing, and so on. Furthermore, it is possible that different MVNOs coexist on the same InP and share the infrastructure 
and radio resources, which maximizes the utilization of resources and reduces the capital expense (CapEx) and operation expense (OpEx).

In the virtualization environment of HetNet, it is a critical issue to allocate the virtual resources to users in an efficient way. Substantial efforts have been done to research the virtual resource allocation model [8-11]. However, these papers mainly focus on the virtual resource allocation with the assumption that the system is in a steady state; that is, the system has reached its equilibrium. In a system with limited resources, it will take a certain period of time to find the equilibrium sharing the virtual resources into MVNOs to optimize the system performance. As a result, the analysis through steady state based method may not be sufficient which ignores the transient dynamics to reach equilibrium of system. More specifically, it is helpful for making more effective approaches (e.g., interference management, base station sleeping, etc.) to promote system performance if we get the users' behaviors or dynamics. Moreover, most of the existing works aim to maximize the system performance (e.g., utility, throughput, etc.) but do not consider the fairness for MVNOs.

Biological approaches are regarded as an effective method to analyze the time dynamic behaviors of heterogeneous system and some works have been done in this area [12]. In [13], the evolutionary game model was utilized to study the robust equilibrium and the dynamics in wireless networks. However, in $[14,15]$, the dynamics of cognitive radio networks were studied, respectively, using predator-prey model and evolutionary game model.

However, most of these works mainly focused on analyzing the dynamics and fairness in resource allocation procedure, ignoring the system utility. To the best of our knowledge, the dynamic-aware virtual radio resource allocation for MVNOs in virtualization based HetNet considering utility and fairness has not been studied in previous works.

In this paper, we investigate the virtual radio resource allocation problem for virtualized HetNet based on the biological Lotka-Volterra model $[16,17]$ with consideration of user dynamics, fairness for MVNOs, and system utility. In the proposed architecture, users of different MVNOs utilize the virtual radio resources in the resource pool in the virtualization based HetNet system. Users of MVNOs benefit from occupying the virtual radio resources and the population of virtual radio resources increases after users release resources. As a result, the relations among different MVNOs and the virtual radio resources are similar to the resource competing of species in a natural environment. The virtual radio resources and users from different MVNOs can be considered as the environment resources and species in natural systems.

The main contributions of this paper are summarized as follows:

(i) We formulate the virtual radio resource allocation problem in virtualization based HetNet as a population competing model, where the users in the system are considered as the predators in nature environment, and virtual radio resources are preys to users. Users of different MVNOs compete for virtual radio resources from the virtual resource pool.

(ii) We introduce the aggregated utility function into the Lotka-Volterra model and take the fairness and utility into consideration at the same time. Furthermore, a virtual resource allocation algorithm based on LotkaVolterra model is developed in the proposed virtual radio resource management framework.

(iii) The proposed algorithm can quickly capture the time dynamics of system. It is helpful to investigate the behaviors and dynamics of users through the trace of time.

(iv) Simulations are conducted to demonstrate that the proposed virtual resource allocation algorithm outperforms the existing centralized maximal utility (MU) approach, achieving a good trade-off between total utility and fairness.

The rest of this paper is organized as follows. Sections 2 and 3 present the system model and the problem formulation. Section 4 provides the proposed virtual radio resource allocation algorithm in virtualization based HetNet. Analysis of system equilibrium state in the proposed system is presented in Section 5, while in Section 6, performance of the proposed algorithm is evaluated by simulations. Finally, Section 7 concludes the paper.

\section{System Model}

In this section, we will introduce the wireless virtualization in heterogeneous network and the classic Lotka-Volterra model in ecosystem. We will then propose a model of virtual radio resource allocation based on Lotka-Volterra in HetNet.

2.1. Wireless Virtualization in HetNet. Similar to the network virtualization, wireless virtualization needs physical resources to be abstracted into a number of virtual resources. All the virtual resources are in a virtual resource pool which can be utilized by different service providers. In the virtualization based HetNet system, heterogeneous physical radio resources owned by different Infrastructure Providers (InPs) can be abstracted and sliced into virtual wireless resources which can be shared by multiple mobile virtual network operators (MVNOs). The virtual radio resource management framework in virtualized heterogeneous networks is as shown in Figure 1.

The modules and their functions are described as follows:

(1) InPs: The InPs consist of heterogeneous wireless networks (e.g., macro and small cell base stations). InPs own the physical wireless network infrastructure resources and physical radio resources. They can provide these physical radio resources for MVNOs and get revenue.

(2) Hypervisor: The hypervisor virtualizes the physical resources from different InPs and enables the sharing for MVNOs. The allocation and mapping of the virtual resources are realized by Virtual Resource Management and Virtual Resource Mapping modules. Also, the hypervisor is responsible for collecting the users' information from MVNOs. 


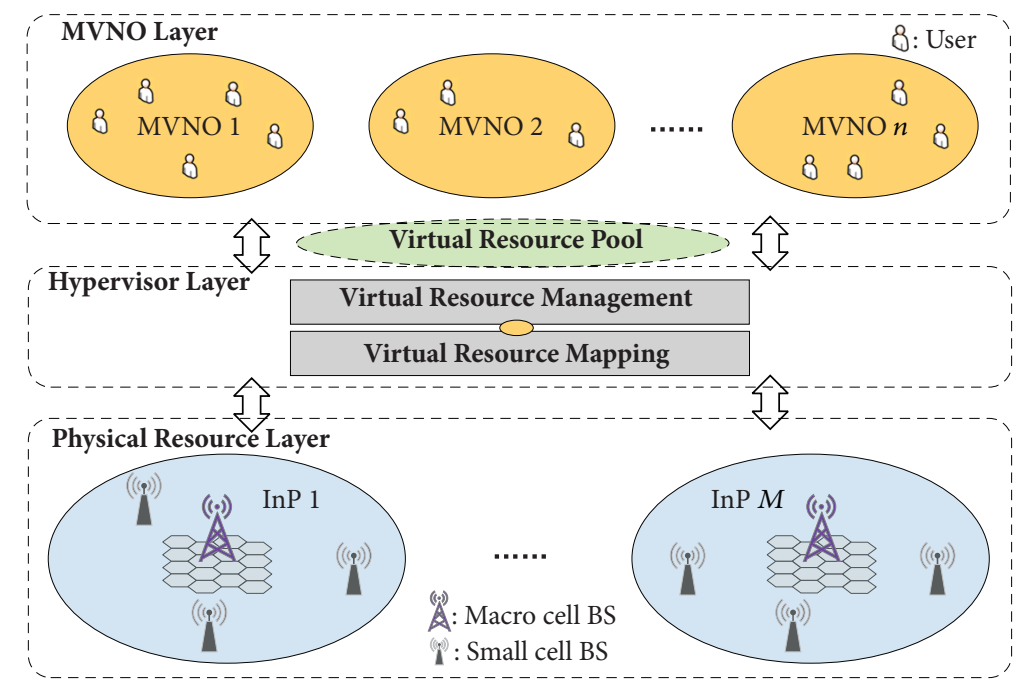

FIGURE 1: The virtual radio resource management framework in virtualized HetNet.

(3) MVNOs: MVNOs lease the physical radio resources from InPs, abstract them into virtual radio resources based on the requests from users, and assign the virtual radio resources to each user. As a result, the MVNOs can provide various services to their subscribers through the same substrate networks.

The virtualization technology can enable the resource sharing in heterogeneous wireless networks which will reduce the CapEx and OpEx. The authors of [18] have estimated that 40 percent of 60 billion USD may be saved using the virtualization technology in wireless networks. In the virtualization based heterogeneous networks, a significant issue is how to catch the time dynamics of users and allocate virtual radio resources into multiple MVNOs which is the purpose of this paper.

2.2. The Lotka-Volterra Model. The Lotka-Volterra model is a mathematical population model of biology which was developed by Alfred J. Lotka (1925) [19] and Vito Volterra (1926) [20]. Classic Lotka-Volterra competition model describes relationship and dynamics of different animal populations competing for shared resources. Assuming that $x_{1}$ and $x_{2}$ represent populations of two species and all the parameters in this model are positive, then

$$
\begin{aligned}
& \dot{x}_{1}=r_{1} x_{1}\left(1-\frac{x_{1}}{K_{1}}-x_{2} \frac{\alpha_{1}}{K_{1}}\right) \\
& \dot{x}_{2}=r_{2} x_{2}\left(1-\frac{x_{2}}{K_{2}}-x_{1} \frac{\alpha_{2}}{K_{2}}\right)
\end{aligned}
$$

The parameters $r_{1}$ and $r_{2}$, respectively, refer to the intrinsic growth rate of the two species, $K_{1}$ and $K_{2}$ are the carrying capacity of the two species, and $\alpha_{1}$ and $\alpha_{2}$ are the inter-specific competition coefficient which reflects that the individuals of one species have inhibited influence on the competitors of the other species. In this model, for example, $\alpha_{1}$ denotes the inhibited effect the individual of species $x_{1}$ has on $x_{2}$, that is, the resource each individual of species $x_{2}$ occupies equal to that $\alpha_{1}$ individual of species $x_{1}$ occupies.

In the Lotka-Volterra model, the population of a species not only depends on the limitation of resources in ecosystem, but also is affected by the survival competing with another species. When a species grows fast, the population of another species will decrease. In the extreme situation, one species can reach its carrying capacity, while the population of another species keeps on the lowest level.

2.3. Proposed Virtual Resource Allocation Model. The resource competing among different species in the natural environment is similar to the virtual radio resource competing in the virtualized wireless networks. In the virtualization based HetNet system, users of different MVNOs utilize the virtual radio resources in the resource pool. Users of MVNOs benefit from occupying the virtual radio resources and the population of virtual radio resources increases after users release resources. Similarly, the population of a MVNO will be limited when other MVNOs occupy too much radio resources (because the radio resources are always limited in wireless system).

Inspired by biological systems and models (i.e., LotkaVolterra model) in nature, we propose an ecology based model for virtual radio resource allocation and users dynamics analysis in HetNet system.

The proposed ecological model in Figure 2 describes the paradigm of virtual resource competing in HetNet, which consists of preys in the environment and $n$ species. The species represent different mobile virtual network operators (MVNOs), while the environment resources are considered as virtual radio resource pool of HetNet. The MVNOs fed on virtual radio resource blocks must continually evolve to ensure sustainability and meet the changing environment, which is analogous to species that have to survive and evolve by consuming environment resources. Users of every MVNO and the virtual radio resources form a "food chain." Just like the biological system, MVNOs and virtual radio resources 


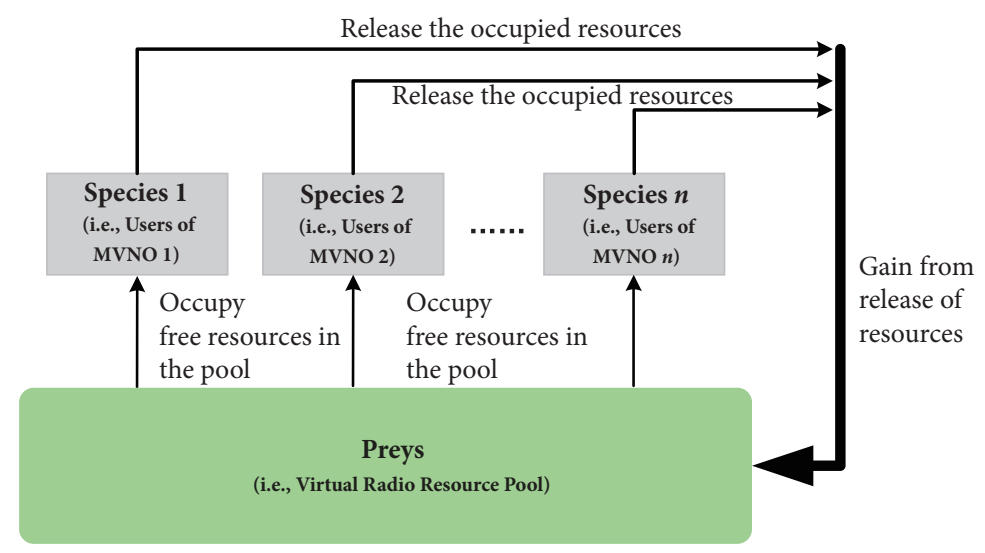

FIgURE 2: The proposed virtual resource allocation model.

can be considered as predators and preys, respectively, the populations of MVNOs benefit from competing to occupy virtual radio resources, and virtual radio resource pool increases when users release the resources after completing communication. However, the total amount of resources in a communication system is limited and fixed, which is different from the common biological system.

Under specific assumptions, the proposed model can be shown to mechanistically represent competition for resources among species. This can be extended into wireless communication system where MVNOs compete for virtual radio resources.

\section{Problem Formulation}

In this section, we formulate the virtual resource allocation problem in virtualized HetNet with the biological model. Firstly, we present the utility functions for users and MVNOs. Then, the virtual radio resource allocation is formulated to resource competing among MVNOs according to the utilities of them.

3.1. Utility Function Definition. In our virtualized wireless networks, users lease virtual resources from their MVNOs. The virtual resources allocated to users are mapped to substrate physical networks. Users pay to their MVNOs based on the data rate they can get. Ensuring the total utility of the system is an important goal of this paper, so that the utility function should firstly be defined. By mapping the utility function with the competing coefficient of biological competing model, we could guarantee that the MVNO with higher aggregated utility can get more resources. As a function of data rate, the utility of user $j$ at time $t$ can be mathematically expressed by [21]:

$$
u_{j, t}=\frac{r_{j, t}{ }^{1-\beta_{j}}}{1-\beta_{j}} \quad\left(0<\beta_{j}<1\right)
$$

where $r_{j, t}$ is the potential data rate of user's virtual resource request at time $t$ and $\beta_{j}$ represents the traffic type of user request $j$. The $r_{j, t}$ can be derived by the Shannon formula based on the bandwidth (denoted as $b_{j, t}$ ) required by the user request $j$ and the effective signal-to-interference-plus-noise ratio at time $t\left(\right.$ denoted as $\left.S I N R_{j, t}\right)$ :

$$
r_{j, t}=b_{j, t} \cdot \log _{2}\left(1+S I N R_{j, t}\right)
$$

We assume that utilities of virtual resource requests are positive and virtual radio resources are allocated periodically every $T$ time so that time index $t$ can be ignored. The utility function of user $j$ can be reformulated as

$$
u_{j}=\frac{\left[b_{j} \cdot \log _{2}\left(1+\operatorname{SINR} R_{j}\right)\right]^{1-\beta_{j}}}{1-\beta_{j}} \quad\left(\quad 0<\beta_{j}<1, b_{j} \geq 0\right)
$$

Then, we can obtain the aggregated utility of MVNO $i$,

$$
U_{i}=\sum_{j} u_{j}=\sum_{j} \frac{\left[b_{j} \cdot \log _{2}\left(1+\operatorname{SINR}_{j}\right)\right]^{1-\beta_{j}}}{1-\beta_{j}}
$$

subject to

$$
\begin{aligned}
\sum_{j} b_{j} & \leq B_{\text {total }} \\
0 & <\beta_{j}<1 \\
b_{j} & \geq 0
\end{aligned}
$$

where $B_{\text {total }}$ is the total bandwidth of the system.

\subsection{Proposed Virtual Resource Allocation Problem Formula-} tion. In the proposed virtualized wireless HetNet, MVNOs need to compete for virtual resources according to the requests of their users. The objective of this paper is to develop a virtual resource allocation algorithm improving the total utility of system and catching the time dynamics of users which is important for network management.

The population model of every MVNO can be expressed by a system of Ordinary Differential Equations (ODEs) which are powerful tools for modelling dynamic systems that 


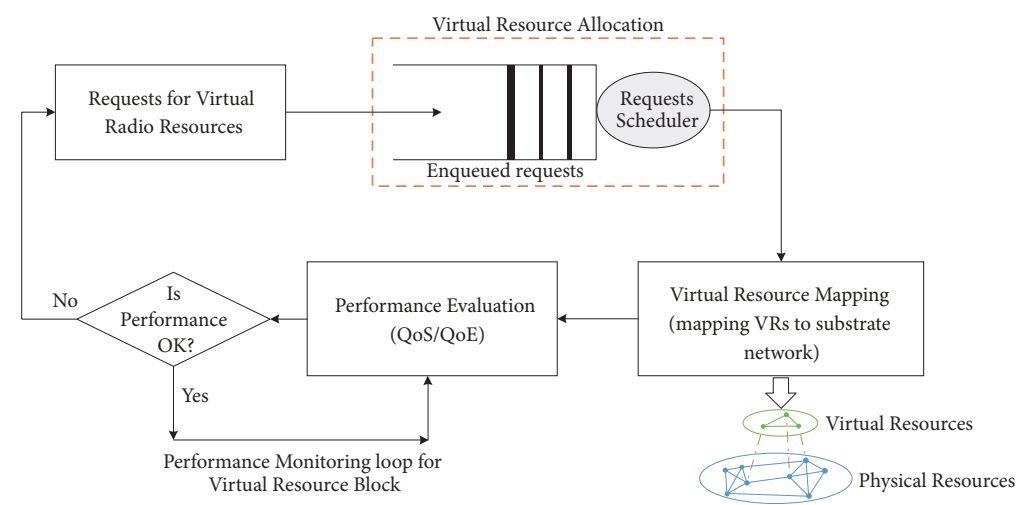

FIGURE 3: The virtual radio resource allocation process in HetNet.

change with time $(\mathrm{d} / \mathrm{dt})$. The population (denoted as $N_{i}(t)$ ) is defined as the number of users who are using the virtual radio resources without interruption in MVNO $i$ at time $t$. The model can be expressed as

$$
\dot{N}_{i}=g_{i} N_{i}\left[1-\frac{1}{K_{i}} N_{i}-\frac{\alpha_{i}}{K_{i}}\left(\sum_{p=1, p \neq i}^{n} N_{p}\right)\right]
$$

subject to

$$
\begin{aligned}
g_{i} & >0, \alpha_{i}>0 \\
N_{i}, K_{i} & \in \mathbb{N}^{+} \\
N_{i} & \leq K_{i}
\end{aligned}
$$

where $g_{i}$ is the intrinsic growth rate of users occupying the virtual radio resource, while $K_{i}$ represents carrying capacity of the system in terms of MVNO $i$. In this model, $\alpha_{i}$ represents the average competition coefficient of other MVNOs on MVNO $i$, and the term $\alpha_{i}\left(\sum_{p=1, p \neq i}^{n} N_{p}\right)$ can be thought of as the decrease in growth rate of MVNO $i$ due to the presence of other MVNOs, so that $N_{i} / K_{i}$ and $\left(\alpha_{i} / K_{i}\right)\left(\sum_{p=1, p \neq i}^{n} N_{p}\right)$ can be, respectively, seen as the virtual radio resources already occupied by MVNO $i$ and other MVNOs.

To ensure utility of the whole system, we define the competition coefficient of virtual network $i$ associated with the aggregated utility. Firstly, we assume that the competing coefficient $\alpha_{i}$ is inversely associated with the aggregated utility $U_{i}$ so that the MVNO with higher aggregated utility will get more resources. Then, we introduce the adjustment factor $a_{i}$ to make sure that the MVNO with higher priority can get lower competing coefficient, and as a result the priorities of some MVNOs can be guaranteed. So, we define the competing coefficient of virtual network $i$ as $\alpha_{i}=a_{i}+$ c . $\left(1 / U_{i}\right)$ (c is a constant to make sure the values of $\alpha_{i}$ satisfy the conditions of convergence). Now we can derive the differential equations of population density as

$$
\dot{N}_{i}=g_{i} N_{i}\left\{1-\frac{1}{K_{i}} N_{i}-\frac{a_{i}+c \sum_{j}\left(\left(1-\beta_{j}\right) /\left[b_{j} \cdot \log _{2}\left(1+\operatorname{SINR} R_{j}\right)\right]^{1-\beta_{j}}\right)}{K_{i}}\left(\sum_{p=1, p \neq i}^{n} N_{p}\right)\right\}
$$

The MVNO with bigger potential aggregated utility may occupy more virtual resource block because it has lower average competition rate with other populations. In each MVNO, virtual resource blocks in the resource pool are assigned to users according to their potential utility $u_{j}$. And generally, fairness and priorities of some virtual networks can be guaranteed via adjusting the variable $a_{i}$. On the one hand, we can set a minimum value of $a_{i}$ for every virtual network so that the coexistence and fairness are maintained. On the other hand, we can set a bigger value for a MVNO with priority to guarantee that it can occupy more resources even when it has lower utility than others.

\section{The Virtual Radio Resource Allocation Algorithm}

In this section, we first present the virtual radio resource allocation progress in virtualization based HetNet and then propose a virtual radio resource allocation algorithm based on the biological competing model.

The system-theoretical model for virtual radio resource allocation progress is as shown in Figure 3. Users request virtual radio resources from their MVNOs and get corresponding services. The requests for virtual radio resources arrive in real time. Then MVNO will conduct the virtual resource allocation for its users. Firstly, the requests will be enqueued at MVNO's master queue according to their priorities and arrival time. Then they will be scheduled to occupy the resources every $T$ time based on their requirement and service level agreement. This procedure is called virtual resource allocation which is investigated in this paper. Then, the virtual resource mapping is conducted, and after the virtual resources are mapped to substrate physical networks, users can start the data transmission procedures. Closed performance monitoring loop can be adopted to evaluate 


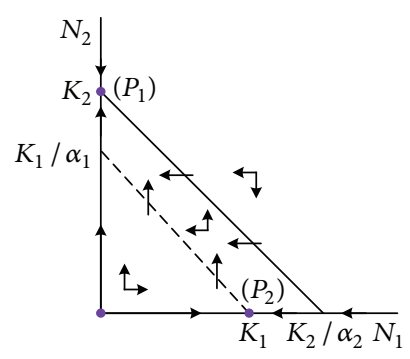

(a)

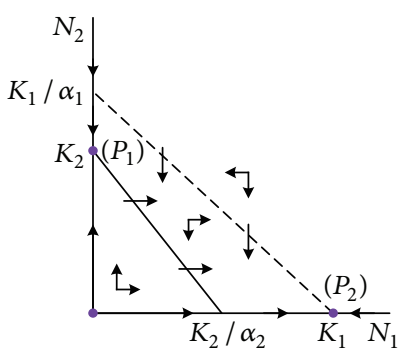

(b)

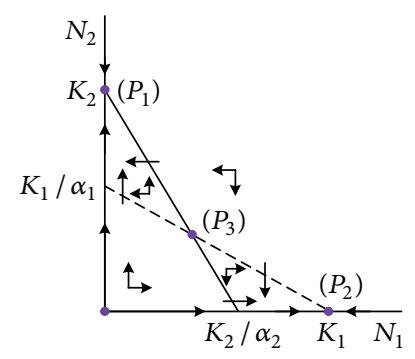

(c)

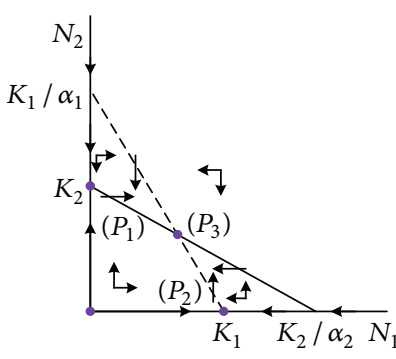

(d)

FIGURE 4: The isocline cases with steady states and flow patterns for the proposed model. Thick dashed lines indicate $N_{1}$-isoclines; thick solid lines represent $\mathrm{N}_{2}$-isoclines.

the performance dynamically. If the performance could not satisfy user's requirement (because the physical channel is changing dynamically), this request will be scheduled in the next period of time.

The proposed model is then applied to virtual radio resource allocation in heterogeneous wireless networks. In the proposed model, the MVNO has bigger value of $\alpha$ and can occupy less resources because other populations have more inhibited influence on it. The model can guarantee the coexistence of all MVNOs with stability and fairness. Thus we can dynamically allocate the virtual radio resources in the pool of HetNet by adjusting the value of $\alpha$ and maintaining the competing and coexisting relationship among MVNOs. We assume that all resource allocation is synchronously performed every $T$ time. Each MVNO gets virtual resources based on its competition coefficient $\alpha_{i}$ which is a function of aggregated utility of MVNO $i$. MVNOs are able to manage the virtual resources and allocate them to users according to their aggregated utility and users' demands. The competition coefficient of each MVNO can also be adjusted based on the knowledge and user dynamics of the whole system to get a more efficient utilizing of resources. The details of virtual radio resource allocation algorithm are described as in Algorithm 1.

\section{Analysis of System Equilibrium State in the Proposed System}

In this section, we present the analysis of system equilibrium state from the aspects of existence, type, and stability.

5.1. The Existence and Category of Equilibrium Points. The important issue regarding system dynamics is the equilibrium of the model. In order to simplify the problem, let us discuss the system which consists of two MVNOs. We could get an algebraic equation set with the right sides of the OEDs (7):

$$
\begin{aligned}
& g_{1} N_{1}\left(1-\frac{1}{K_{1}} N_{1}-\frac{\alpha_{1}}{K_{1}} N_{2}\right)=0 \\
& g_{2} N_{2}\left(1-\frac{1}{K_{2}} N_{2}-\frac{\alpha_{2}}{K_{2}} N_{1}\right)=0
\end{aligned}
$$

As known in mathematics, the real roots of this algebraic equation set are called the equilibrium point of the model.
For equilibrium point $P_{0}\left(N_{1}{ }^{0}, N_{2}{ }^{0}\right)$, if the solutions of ODEs always meet the restrictions $\lim _{\mathrm{t} \rightarrow \infty} N_{1}(\mathrm{t})=N_{1}{ }^{0}$ and $\lim _{\mathrm{t} \rightarrow \infty} N_{2}(\mathrm{t})=N_{2}{ }^{0}$ at any initial condition, we call $P_{0}$ a stable equilibrium point (asymptotic stability); otherwise $P_{0}$ is unstable (not asymptotic stability). Obviously, there are two equilibrium points $P_{1}=\left(0, K_{2}\right)$ and $P_{2}=\left(K_{1}, 0\right)$ in addition to the trivial equilibrium point $N_{1}=N_{2}=0$ for the set of ODEs (7) $(n=2)$. When the algebraic equation set (13) has a nonnegative solution $N_{1}=N_{1}{ }^{*}, N_{2}=N_{2}{ }^{*}$, we find the third equilibrium point of the system, that is, $P_{3}\left(N_{1}{ }^{*}, N_{2}{ }^{*}\right)$.

To analyze the properties of this system, phase plane analysis of the model equations is carried out. Firstly, we plot the zero growth rate line for each species taking $N_{1}$ and $\mathrm{N}_{2}$ (number of users occupying resources in MVNOs 1 and 2) as the coordinate. The zero growth line can be obtained by drawing a line between $N_{1}$ and $N_{2}$ intercepts of each species. Users in both species will increase until they reach the zero growth isocline. The MVNO represented on the abscissa meets the isocline horizontally, and the one represented on the ordinate will do it vertically. Figure 4 illustrates the flow patterns and four possible types of relations between the isoclines of MVNOs 1 and 2. From the flows across the isoclines, the direction fields within each separate region in the phase plane can be worked out. For example, in case (a), it is obvious within the area bounded by these two isoclines that the flow is towards the top-left corner, which implies that all solutions will tend to the $N_{2}$-only state $\left(N_{1}, N_{2}\right)=\left(0, K_{2}\right)$.

Hence, we can find three equilibrium points for the proposed model (excluding the trivial equilibrium point): $P_{1}=\left(0, K_{2}\right), P_{2}=\left(K_{1}, 0\right), P_{3}=\left(N_{1}{ }^{*}, N_{2}{ }^{*}\right)=\left(\left(K_{1}-\right.\right.$ $\left.\left.\alpha_{1} K_{2}\right) /\left(1-\alpha_{1} \alpha_{2}\right),\left(K_{2}-\alpha_{2} K_{1}\right) /\left(1-\alpha_{1} \alpha_{2}\right)\right)$. In the $N_{2}$-only state, species 2 reaches its carrying capacity and species 1 goes extinct, while in the $N_{1}$-only state, species 2 goes extinct and species 1 tends to its carrying capacity. In cases (c) and (d), there is a coexistence state in which both species have nonzero abundance.

5.2. The Stability of Equilibrium Points. Now, we analyze the stability of equilibrium points. Since the proposed model is applied to wireless communication system and the users of each MVNO cannot be decreased to zero considering the revenue of MVNOs, we need not to consider equilibrium points $P_{1}=\left(0, K_{2}\right)$ and $P_{2}=\left(K_{1}, 0\right)$. Furthermore, wireless communication systems should be guaranteed the asymptotic 


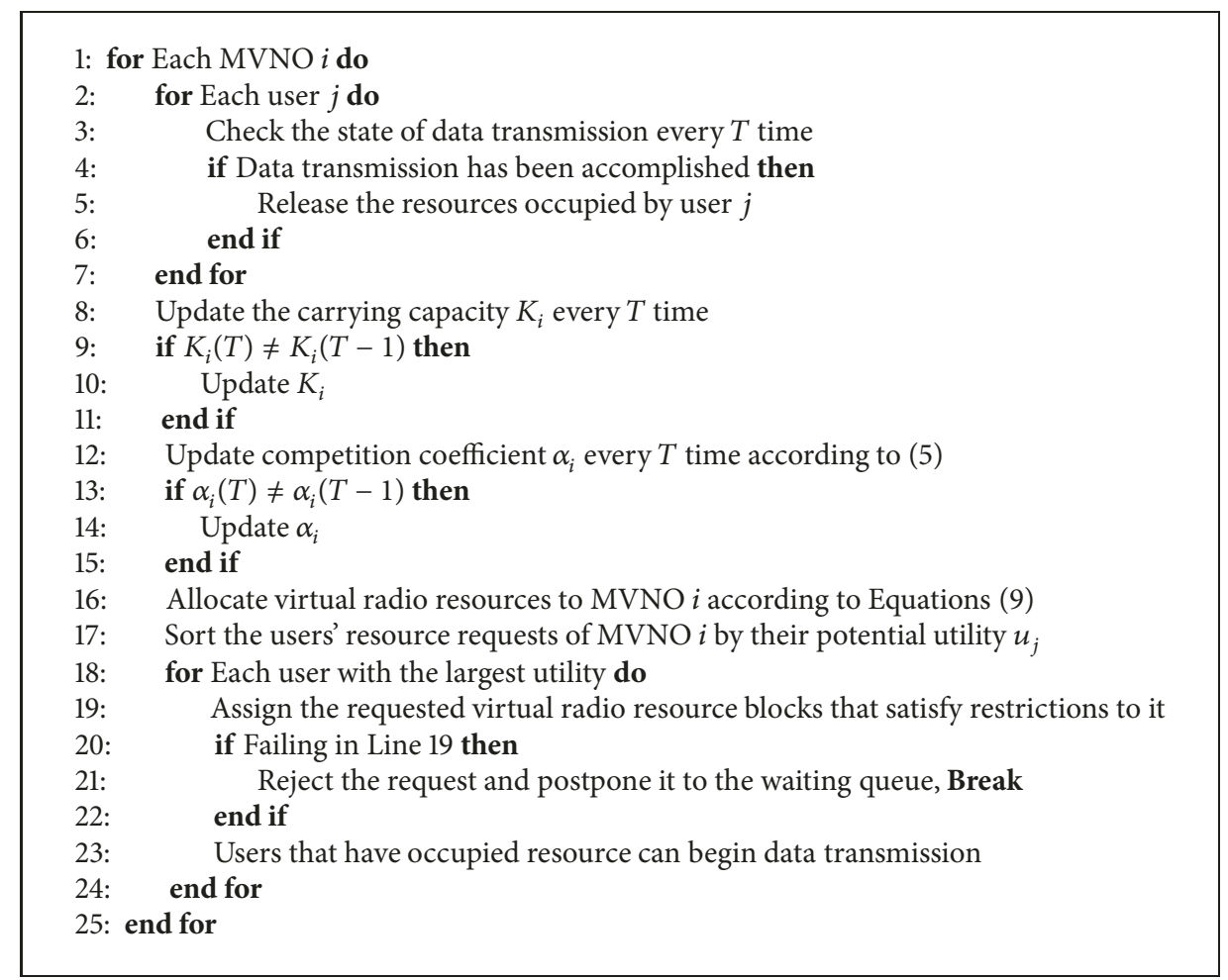

Algorithm 1: Biological Competing Resource Allocation Algorithm.

stability, so that it is necessary to make sure whether the equilibrium point $P_{3}\left(N_{1}{ }^{*}, N_{2}{ }^{*}\right)$ is stable. Firstly, we give a proposition about the stability of equilibrium points of (7) $(n=2)$.

Proposition 1. If the two inequalities $K_{2}<K_{1} / \alpha_{1}, K_{1}<$ $K_{2} / \alpha_{2}$ are satisfied, the system has three equilibrium points $P_{1}=\left(0, K_{2}\right), P_{2}=\left(K_{1}, 0\right), P_{3}\left(N_{1}{ }^{*}, N_{2}{ }^{*}\right)$, and $P_{3}\left(N_{1}{ }^{*}, N_{2}{ }^{*}\right)$ is the stable equilibrium point. The two MVNOs could coexist in the course of time, $N_{1}(t) \longrightarrow N_{1}{ }^{*}, N_{2}(t) \longrightarrow N_{2}{ }^{*}$.

Proof. Because $P_{3}\left(N_{1}{ }^{*}, N_{2}{ }^{*}\right)$ is the equilibrium point, the linearization of the ODEs $(7)(n=2)$ near point $P_{3}$ can be expressed as

$$
\begin{aligned}
& \frac{d\left(N_{1}-N_{1}{ }^{*}\right)}{d t} \\
& =g_{1} N_{1}{ }^{*}\left(-\frac{1}{K_{1}}\left(N_{1}-N_{1}{ }^{*}\right)-\frac{\alpha_{1}}{K_{1}}\left(N_{2}-N_{2}{ }^{*}\right)\right) \\
& \frac{d\left(N_{2}-N_{2}{ }^{*}\right)}{d t} \\
& =g_{2} N_{2}{ }^{*}\left(-\frac{1}{K_{2}}\left(N_{1}-N_{1}{ }^{*}\right)-\frac{\alpha_{2}}{K_{2}}\left(N_{2}-N_{2}{ }^{*}\right)\right)
\end{aligned}
$$
is

Then, the characteristic equation of the coefficient matrix

$$
\left|\begin{array}{cc}
\lambda+\frac{g_{1}}{K_{1}} N_{1}{ }^{*} & \frac{g_{1} \alpha_{1}}{K_{1}} N_{1}{ }^{*} \\
\frac{g_{2} \alpha_{2}}{K_{2}} N_{2}{ }^{*} & \lambda+\frac{g_{2}}{K_{2}} N_{2}{ }^{*}
\end{array}\right|=0
$$

That is,

$$
\lambda^{2}+\left(\frac{g_{1}}{K_{1}} N_{1}^{*}+\frac{g_{2}}{K_{2}} N_{2}{ }^{*}\right) \lambda+\Delta N_{1}^{*} N_{2}^{*}=0
$$

where $\Delta=g_{1} g_{2}\left(1-\alpha_{1} \alpha_{2}\right) / K_{1} K_{2}$.

The discriminant of (13) is

$$
\begin{aligned}
& \left(\frac{g_{1}}{K_{1}} N_{1}{ }^{*}+\frac{g_{2}}{K_{2}} N_{2}{ }^{*}\right)^{2}-4 \Delta N_{1}{ }^{*} N_{2}{ }^{*} \\
& =\left(\frac{g_{1}}{K_{1}} N_{1}{ }^{*}-\frac{g_{2}}{K_{2}} N_{2}{ }^{*}\right)^{2}+4 \Delta N_{1}{ }^{*} N_{2}{ }^{*}>0
\end{aligned}
$$

So (13) has two different real eigenvalues: $\lambda_{1}<\lambda_{2}$, and by using the Vieta theorem, we can get

$$
\begin{aligned}
\lambda_{1}+\lambda_{2} & =-\left(\frac{g_{1}}{K_{1}} N_{1}{ }^{*}+\frac{g_{2}}{K_{2}} N_{2}{ }^{*}\right) \\
\lambda_{1} \lambda_{2} & =\Delta N_{1}{ }^{*} N_{2}{ }^{*}
\end{aligned}
$$

because $K_{2}<K_{1} / \alpha_{1}$ and $K_{1}<K_{2} / \alpha_{2}$ are satisfied, just as the situation in Figure 4(d), that is, $g_{2} K_{1} / g_{1} K_{2} \alpha_{1}>g_{2} / g_{1}>$ $g_{2} K_{1} \alpha_{2} / g_{1} K_{2}$. As a result, $g_{2} K_{1} / g_{1} K_{2} \alpha_{1}-g_{2} K_{1} \alpha_{2} / g_{1} K_{2}=$ $g_{2} K_{1}\left(1-\alpha_{1} \alpha_{2}\right) / g_{1} K_{2} \alpha_{1}>0$, so that $\Delta>0$. We can get that $\lambda_{1}<0$ and $\lambda_{1}<0$ by putting $\Delta>0$ into (15). Thus, the equilibrium point $P_{3}\left(N_{1}{ }^{*}, N_{2}{ }^{*}\right)$ is stable (asymptotic stability).

Similarly, we can prove that $P_{3}\left(N_{1}{ }^{*}, N_{2}{ }^{*}\right)$ is not stable when $K_{2}>K_{1} / \alpha_{1}$ and $K_{1}>K_{2} / \alpha_{2}$ are satisfied which is illustrated in Figure 4(c). Actually, in case (c), MVNO 


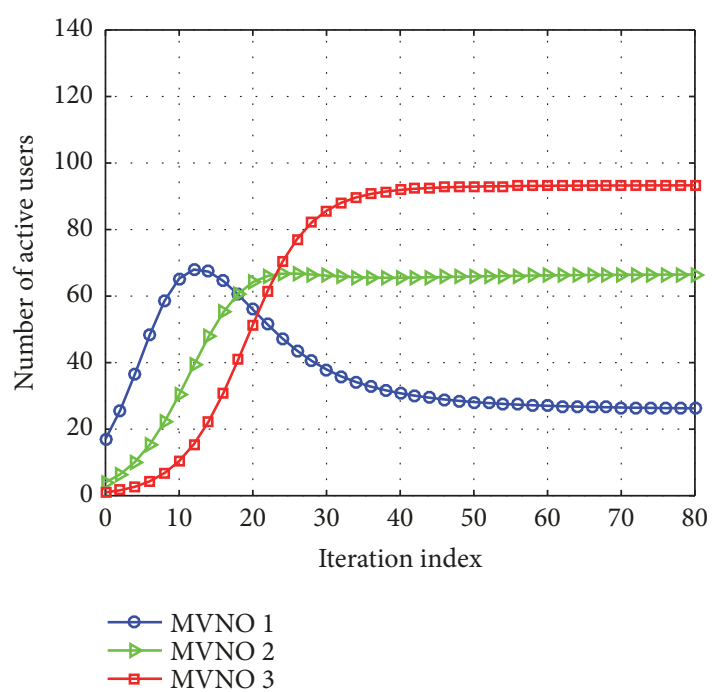

Figure 5: The population dynamics of MVNOs in the proposed system $(n=3)$.

1 can outcompete MVNO 2, but MVNO 2 can also outcompete MVNO 1. The equilibrium point $P_{3}$ may evolve to $P_{1}$ or $P_{2}$ which depends on the initial conditions of the system.

Hence, we could guarantee the coexistence of MVNOs over time by adjusting the values of system parameters every $T$ time based on the proposition and make sure that the proposed system can reach a stable equilibrium.

\section{Simulation Results and Discussion}

In this section, simulation results are given to evaluate the performance of the proposed algorithm. In the simulation, the parameters are designed to model a high-loaded system where the available resources are not enough to serve all the users. We assume that there are three MVNOs in the system, and the virtual resource blocks for different user requests in MVNOs have the different bandwidths, which are with uniform distribution. The following parameters are used: $a_{1}=$ $0.08, a_{2}=0.05, a_{3}=0.03, \mathrm{c}=1.6 \times 10^{5}, \beta_{j}=0.5, b_{j} \sim U(0,4)$ $\mathrm{MHz}, S_{I N R} \sim U(5,20) \mathrm{dB}$. The three MVNOs are assumed to have the different number of users $(J)$ at time $t$.

Figure 5 illustrates the population dynamics for the proposed system which has three MVNOs $(n=3)$. The convergence of Algorithm 1 is evaluated with resource growth rates $g_{i}=0.3$, number of users in each MVNO $J_{1}=100$, $J_{2}=110, J_{3}=120$, and carrying capacities $K_{1}=90$, $K_{2}=100, K_{3}=110$. As can be seen in Figure 5, the number of active users in each MVNO converges within 50 iterations. This result, together with the previous analysis, indicates that the proposed Algorithm 1 converges to a stable equilibrium.

It also can be seen from Figure 5 that the MVNO with higher service level (smaller $a_{i}$ ) can get more virtual resources to serve its users, because a smaller $a_{i}$ reduces the competition coefficient, as defined in (9). The MVNO with larger competition coefficient can also get some resources, although the resources are limited in the system. MVNOs

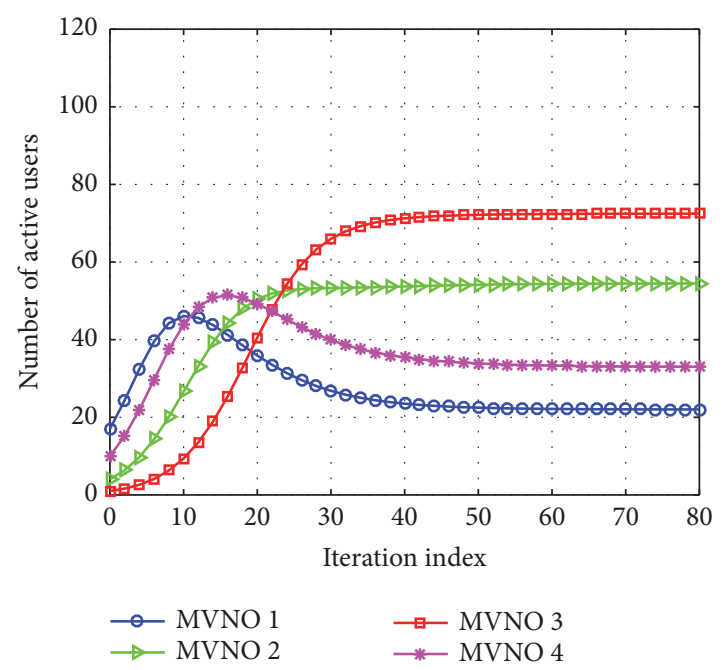

FIgURE 6: The population dynamics of MVNOs in the proposed system $(n=4)$.

coexist in the proposed system and maintain a dynamic balance actually.

In Figure 6, the population dynamics is simulated with four MVNOs to evaluate the convergence of the proposed algorithm when the number of MVNOs scales. As can be seen from Figure 6, the number of active users in each MVNO also converges after iterations. However, the number of MVNOs affects the rate of convergence; more MVNOs may lead to lower convergence rate. For example, the number of active users in MVNO 3 converges within 30 iterations when there are 3 MVNOs in the system, but when there are 4 MVNOs in the system, it converges after 45 iterations. Furthermore, the number of active users of each MVNO will converge to lower level when the system has more MVNOs, because the resources are limited.

As the maximal utility (MU) approach can achieve maximal system utility, it has become a very popular method in virtual resource allocation. For example, the authors in [8] have worked on the virtual resource allocation for MVNOs based on the MU of system. There are also some existing works which focus on the fairness and use the round-robin (RR) method [22]. Therefore, we compare the performance of the proposed Lotka-Volterra solution (LVS) with that of the existing MU approach and RR method.

In Figures 7-9, we compare the utilities of MVNOs when the number of users requesting resource in MVNOs increases from $(50,60,70)$ to $(100,110,120)$, with resource growth rates $g_{i}=0.3$, carrying capacities $K_{1}=90, K_{2}=100, K_{3}=110$.

As can be seen in Figures 7-9, a MVNO with higher service level gets higher utility in MU approach, RR method, and the proposed Algorithm 1. With MU approach, the utility of MVNO 3 increases linearly while the utility of MVNO 1 drops to zero when the users in MVNO 1 are over 80. However, with the proposed algorithm or RR method, the utilities are always positive because each MVNO can get some resources to serve users. Therefore, it is verified that the proposed algorithm and RR method can guarantee 


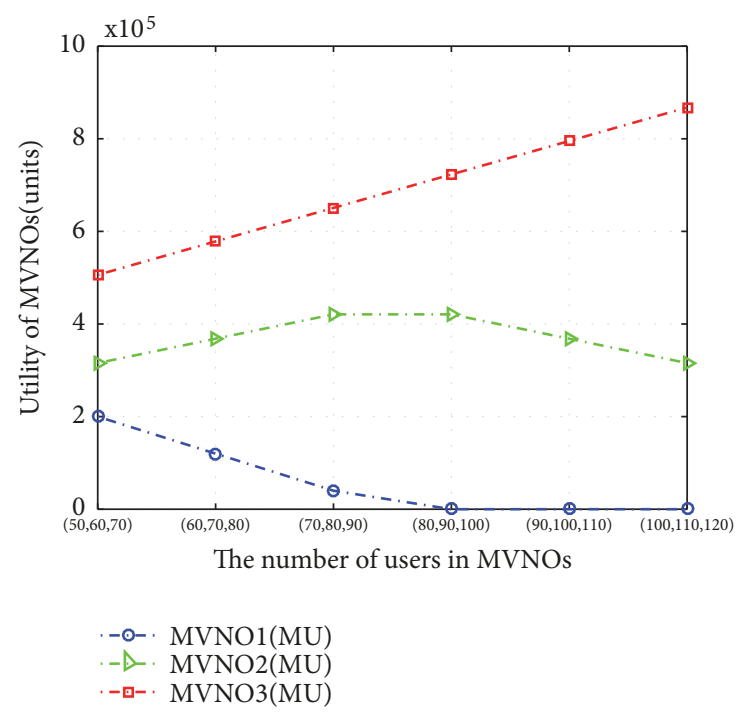

FIGURE 7: Utility of MVNOs in MU approach.

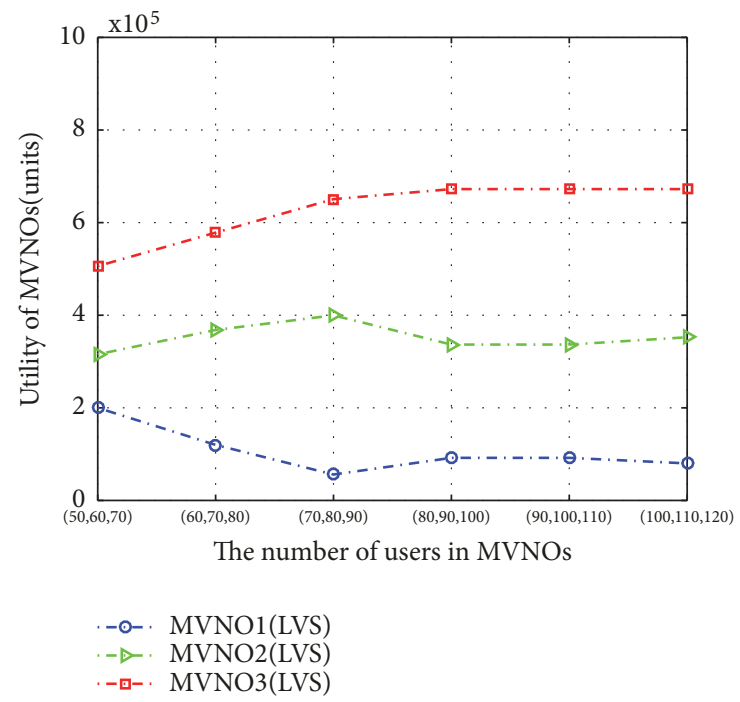

FIGURE 8: Utility of MVNOs in the proposed Algorithm 1.

higher utility for MVNO with lower service level than the MU approach. Also, they improve the fairness in resource allocation process of the system.

To evaluate the fairness of MVNOs in the system, we use the fairness index (FI), which is defined as [23]:

$$
\frac{\left(\sum_{i=1}^{n} N_{i}\right)^{2}}{\left(n\left(\sum_{i=1}^{n}\left(N_{i}\right)^{2}\right)\right)}
$$

The fairness index is widely applied in the literature to evaluate the level of fairness achieved by resource allocation algorithms.

In Figures 10 and 11, we compare the total utility of system and fairness among MVNOs $(n=3)$. As can be seen from Figure 10, the proposed Algorithm 1 achieves higher total utility than RR method, but lower total utility than MU approach when the users in MVNOs are more than $(70,80$,

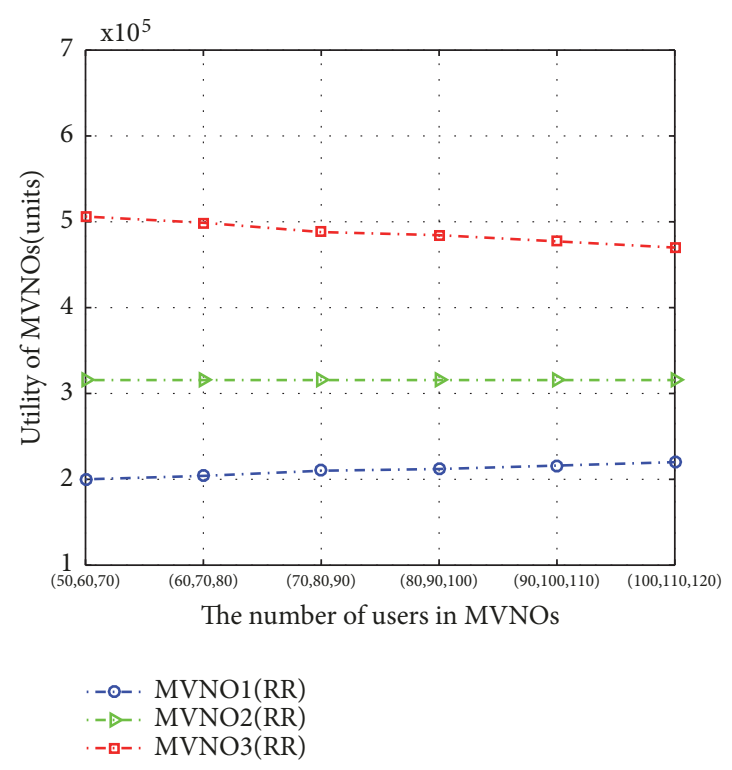

FIgURE 9: Utility of MVNOs in RR method.

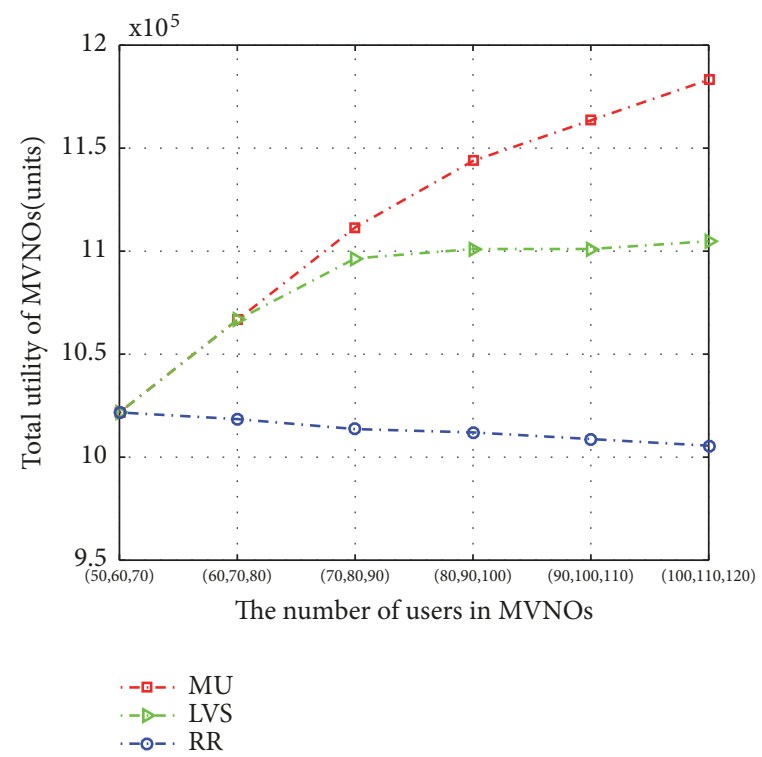

FIGURE 10: Comparison of the total utilities of different approaches.

90). Figure 11 shows that the proposed Algorithm 1 achieves lower fairness index than RR method, but higher fairness index than MU approach when the users in MVNOs are more than $(70,80,90)$. As one can conclude from Figures 10 and 11, the proposed Algorithm 1 can achieve higher fairness index with the cost of small reduction in total utility of system, compared with the MU approach. It achieves a better trade-off between total utility and fairness than the existing methods.

Besides, we could also analyze the time dynamics of users and virtual radio resources in the system by utilizing the Lotka-Volterra model. The users in the system can be considered as the predators in nature environment, and virtual radio resources are preys to users. The number (i.e., 


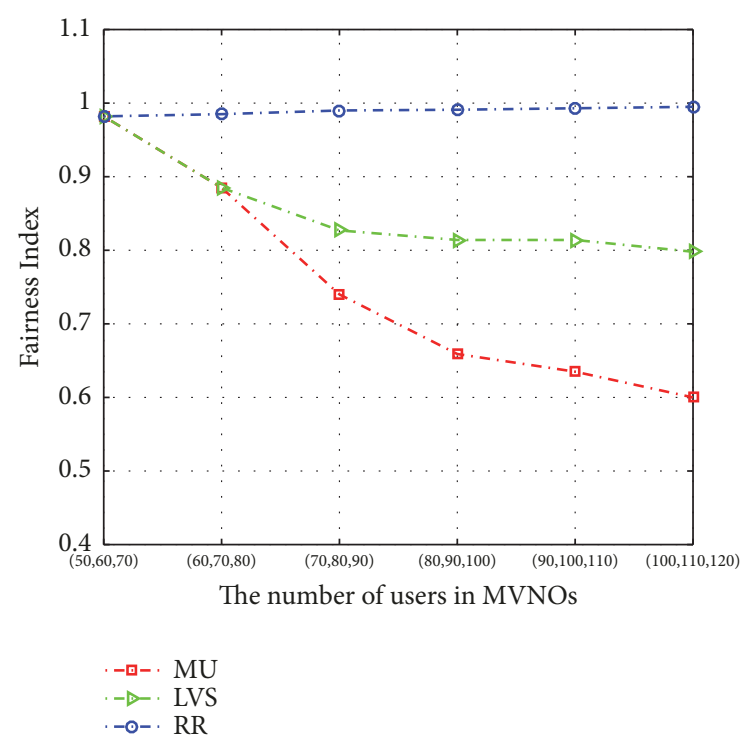

FIgURE 11: Comparison of the fairness index of different approaches.

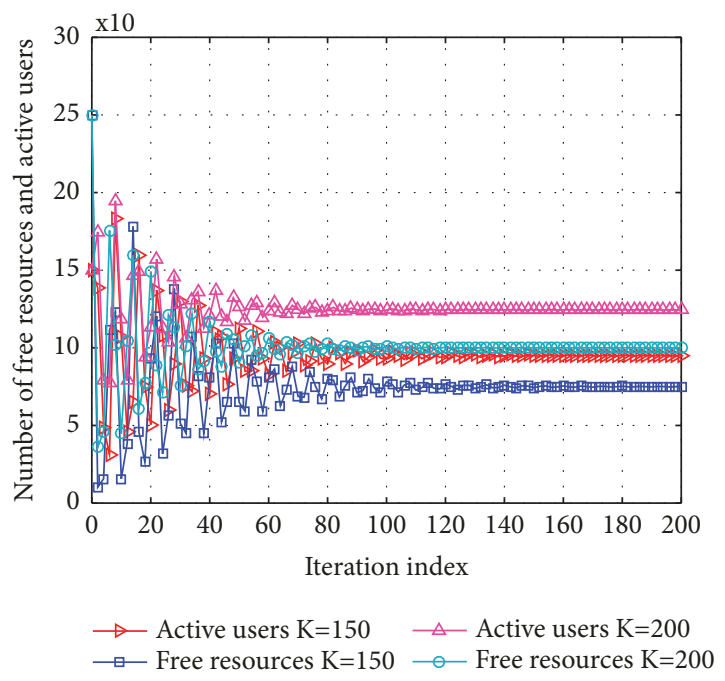

FIGURE 12: The population dynamics of users and virtual radio resource pool in the proposed system. The parameter $K$ is the system capacity.

population) of active users in the system is benefited by the loss of the population of the virtual radio resources. Therefore, the ecological model is helpful to investigate the behaviors and user dynamics of system through the trace of time. The numerical illustrations of the user dynamics are presented in Figures 12 and 13. From Figure 12, we observe that the behaviors of users in the proposed system follow those in the nature environment; that is, the population of resource will decrease when there are too many users in system. The users and virtual radio resources in the system are regulating to each other. Time dynamics of users in different system capacity (i.e., $K=150,200$ ) are simulated, and by adjusting the system capacity $K$ within certain range, we can get an improvement of system throughput, as shown in Figure 12.

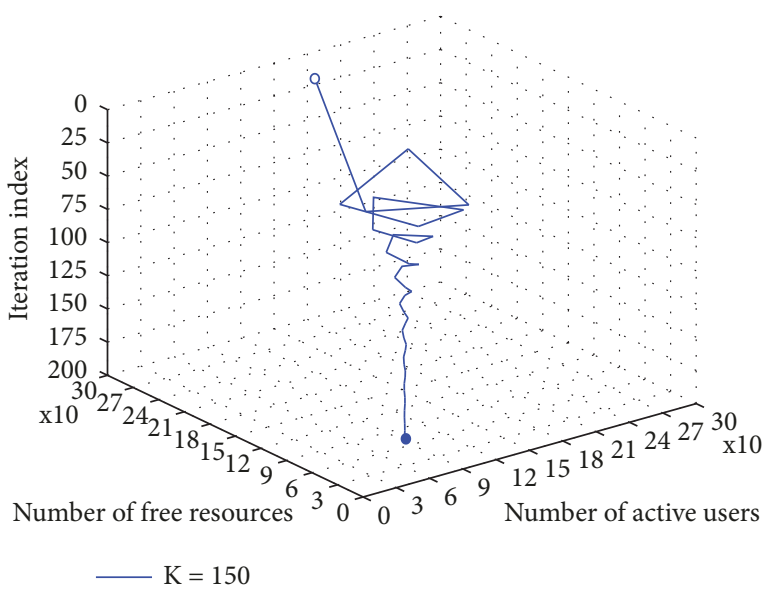

Figure 13: The 3-D phase diagram of the system where users and virtual radio resources are considered predators and preys, respectively.

The phase diagram of user dynamics is illustrated in Figure 13 in which we could catch the dynamics of interaction between users and virtual resources in the system. In this simulation, the system capacity is set as $K=150$. The system converges to its equilibrium after a period of time. As can be seen in these two figures, the dynamics of users and virtual resources can be analyzed utilizing the proposed biological model, and the throughput of system can be improved by increasing the capacity of system.

By understanding the time dynamics of users, we can further develop effective strategies to enhance system performance. For instance, the hypervisor can conduct adaption of system parameters and base station sleeping strategies to improve the channel utilization and energy efficiency.

\section{Conclusion}

In this paper, we have investigated the virtual resource allocation problem in virtualization based heterogeneous wireless networks. The virtual resource allocation problem in HetNet was formulated as a population competing model, where the users in the system are considered as the predators in nature environment, and virtual radio resources are preys to users. Users of different MVNOs compete for virtual radio resources from the virtual resource pool. Accordingly, a virtual resource allocation algorithm based on Lotka-Volterra model was developed, considering system utility, fairness, and dynamics. Simulation results showed that the proposed virtual resource allocation algorithm not only converges within a few iterations, but also achieves a better trade-off between total utility and fairness than existing algorithm. Besides, it can also be utilized to analyze the time dynamics of users which is helpful for making more effective approaches to promote system performance.

\section{Data Availability}

The data used to support the findings of this study are included within the article. 


\section{Conflicts of Interest}

The authors declare that they have no conflicts of interest.

\section{Acknowledgments}

This work is supported by China Ministry of EducationCMCC Research Fund Project no. MCM20160104, National Science and Technology Major Project no. 2018ZX030110004, Beijing Municipal Science and Technology Commission Research Fund Project no. Z171100005217001, and Fundamental Research Funds for Central Universities no. 2018RC06. Besides, we would like to thank EURECOM and OpenAirInterface Software Alliance for their support and help.

\section{References}

[1] C. Liang and F. R. Yu, "Wireless network virtualization: a survey, some research issues and challenges," IEEE Communications Surveys \& Tutorials, vol. 17, no. 1, pp. 358-380, 2015.

[2] M. Kamel, W. Hamouda, and A. Youssef, "Ultra-Dense Networks: A Survey," IEEE Communications Surveys \& Tutorials, vol. 18, no. 4, pp. 2522-2545, 2016.

[3] A. Gudipati, D. Perry, L. E. Li, and S. Katti, "Softran: software defined radio access network," in Proceedings of the 2nd ACM SIGCOMM Workshop on Hot Topics in Software Defined Networking (HotSDN '13), pp. 25-30, August 2013.

[4] Q. Li, R. Q. Hu, Y. Qian, and G. Wu, "Intracell cooperation and resource allocation in a heterogeneous network with relays," IEEE Transactions on Vehicular Technology, vol. 62, no. 4, pp. 1770-1784, 2013.

[5] C. Liang and F. R. Yu, "Wireless virtualization for next generation mobile cellular networks," IEEE Wireless Communications Magazine, vol. 22, no. 1, pp. 61-69, 2015.

[6] A. Blenk, A. Basta, M. Reisslein, and W. Kellerer, "Survey on network virtualization hypervisors for software defined networking," IEEE Communications Surveys \& Tutorials, vol. 18, no. 1, pp. 655-685, 2016.

[7] S. Fan, H. Tian, and W. Wang, "A Radio Resource Virtualization-Based RAT Selection Scheme in Heterogeneous Networks," IEEE Communications Letters, vol. 21, no. 5, pp. 11471150, 2017.

[8] C. Liang, F. R. Yu, H. Yao, and Z. Han, "Virtual Resource Allocation in Information-Centric Wireless Networks with Virtualization," IEEE Transactions on Vehicular Technology, vol. 65, no. 12, pp. 9902-9914, 2016.

[9] T. Leanh, N. H. Tran, D. T. Ngo, and C. S. Hong, "Resource Allocation for Virtualized Wireless Networks with Backhaul Constraints," IEEE Communications Letters, vol. 21, no. 1, pp. 148-151, 2017.

[10] W. H. Chin, Z. Fan, and R. Haines, "Emerging technologies and research challenges for $5 \mathrm{G}$ wireless networks," IEEE Wireless Communications Magazine, vol. 21, no. 2, pp. 106-112, 2014.

[11] D. Kreutz, F. M. V. Ramos, P. E. Verissimo, C. E. Rothenberg, S. Azodolmolky, and S. Uhlig, "Software-defined networking: a comprehensive survey," Proceedings of the IEEE, vol. 103, no. 1, pp. 14-76, 2015.

[12] S. Balasubramaniam, K. Leibnitz, P. Lio, D. Botvich, and M. Murata, "Biological principles for future Internet architecture design," IEEE Communications Magazine, vol. 49, no. 7, pp. 4452, 2011.

[13] H. Tembine, E. Altman, R. El-Azouzi, and Y. Hayel, "Evolutionary games in wireless networks," IEEE Transactions on Systems, Man, and Cybernetics, Part B: Cybernetics, vol. 40, no. 3, pp. 634-646, 2010.

[14] D. Liau, K.-C. Chen, and S.-M. Cheng, "A predator-prey model for dynamics of cognitive radios," IEEE Communications Letters, vol. 17, no. 3, pp. 467-470, 2013.

[15] S.-M. Cheng, P.-Y. Chen, and K.-C. Chen, "Ecology of cognitive radio Ad hoc networks," IEEE Communications Letters, vol. 15, no. 7, pp. 764-766, 2011.

[16] O. T. Solbrig and D. J. Solbrig, Introduction to Population Ecology and Evolution, Addison-Wesley, 1979.

[17] S. B. Hsu, S. P. Hubbell, and P. Waltman, "Competing predators," SIAM Journal on Applied Mathematics, vol. 35, no. 4, pp. 617$625,1978$.

[18] X. Costa-Perez, J. Swetina, T. Guo, R. Mahindra, and S. Rangarajan, "Radio access network virtualization for future mobile carrier networks," IEEE Communications Magazine, vol. 51, no. 7, pp. 27-35, 2013.

[19] A. J. Lotka, Elements of Physical Biology, Williams and Wilkins, 1925.

[20] V. Volterra, "Variations and fluctuations of the number of individuals in animal species living together," Journal du Conseil International pour L'Exploration de la Mer, vol. 3, no. 1, pp. 3-51, 1928.

[21] M. Li, P. N. Tran, D. Wang, and A. Timm-Giel, "Radio resource allocation in LTE using utility functions based on moving average rates," in Proceedings of the 2014 IEEE Wireless Communications and Networking Conference, WCNC 2014, pp. 1891-1896, Turkey, April 2014.

[22] W. Gong, X. Wang, M. Li, and Z. Huang, "Round-robin resource sharing algorithm for device-to-device multicast communications underlying single frequency networks," in Proceedings of the 21st International Conference on Telecommunications, ICT 2014, pp. 191-195, Portugal, May 2014.

[23] H. Zhang, C. Jiang, N. C. Beaulieu, X. Chu, X. Wang, and T. Q. S. Quek, "Resource allocation for cognitive small cell networks: a cooperative bargaining game theoretic approach," IEEE Transactions on Wireless Communications, vol. 14, no. 6, pp. 3481-3493, 2015. 


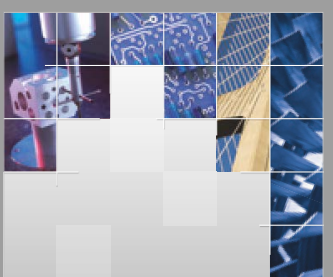

\section{Enfincering}
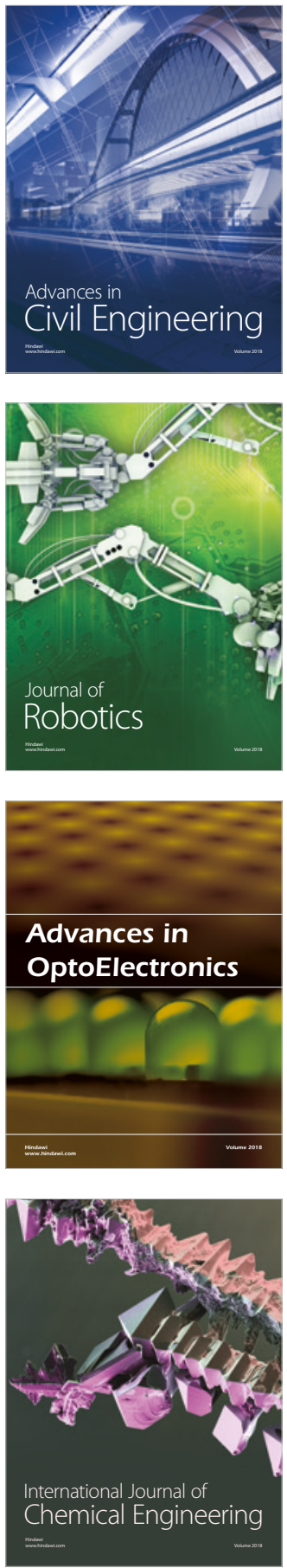

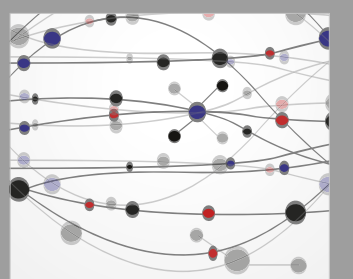

\section{Rotating \\ Machinery}

The Scientific World Journal

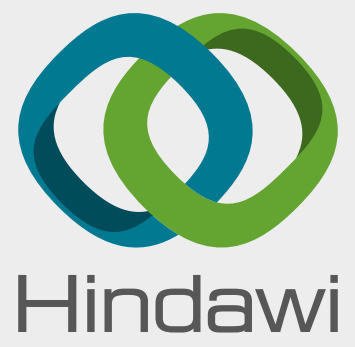

Submit your manuscripts at

www.hindawi.com
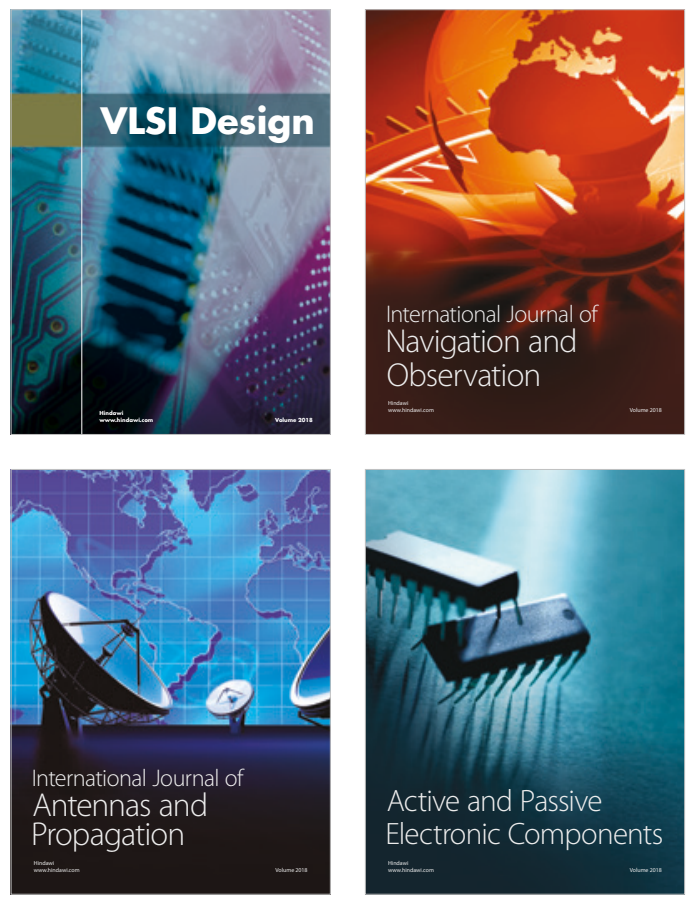
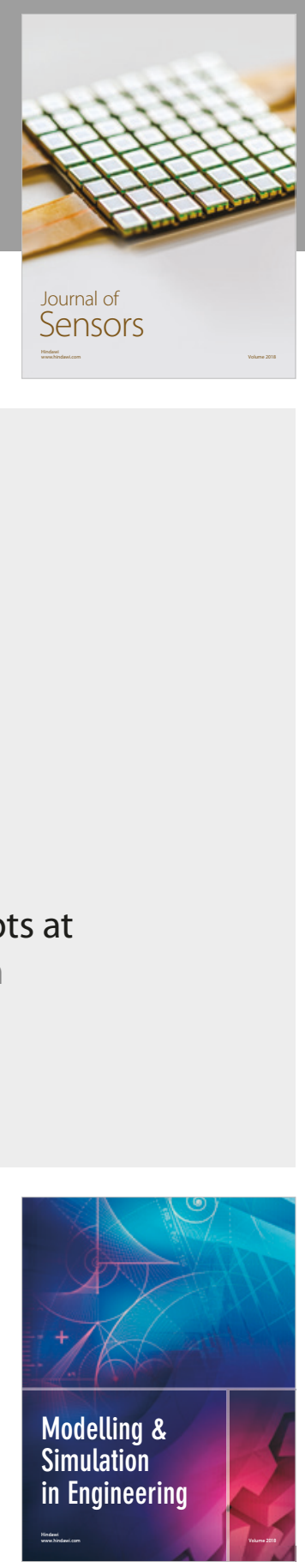

\section{Advances \\ Multimedia}
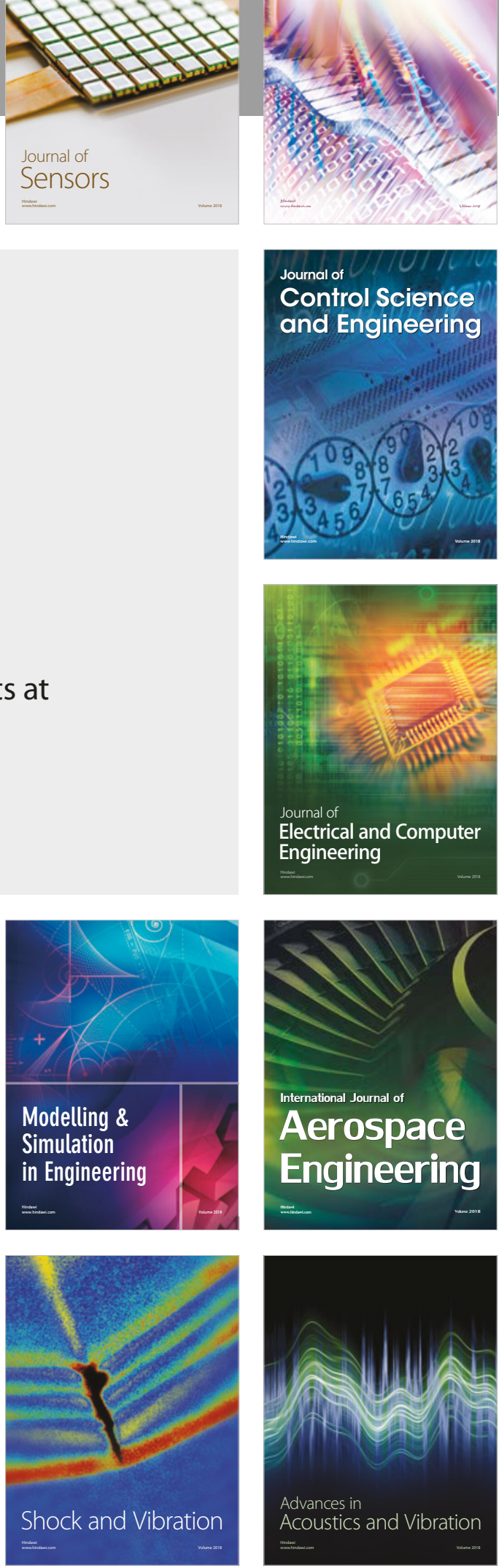